\title{
PREVENCIÓN DE LA TRANSMISIÓN MATERNO-INFANTIL DEL VIH EN GESTANTES Y MADRES AWAJÚN Y WAMPIS DE LA REGIÓN AMAZONAS EN PERÚ
}

\author{
Byelca Huamán ${ }^{1, a}$, Alfonso Gushiken ${ }^{2, b}$, Carlos Benites ${ }^{3, c}$, Fabiola Quiroz ${ }^{4, d}$, Lisset García-Fernández
}

\begin{abstract}
RESUMEN
Objetivos. Identificar las barreras que limitan el cumplimiento de las medidas de prevención de la transmisión maternoinfantil (TMI) del VIH, en comunidades indígenas de la región Amazonas en Perú. Materiales y métodos. Investigación cualitativa con enfoque fenomenológico. Se realizaron entrevistas semiestructuradas a gestantes y madres de niños menores de un año de las comunidades indígenas awajún y wampis diagnosticadas con VIH durante el 2014-2015. Resultados. Participaron 15 de 29 mujeres, siendo el $87 \%$ de la etnia awajún. Se identificaron limitaciones y posibles efectos cuando se impone medidas sanitarias para la prevención de la TMI. Dada la concepción que tienen de las enfermedades, que implica la presencia de síntomas e incapacidad, muchas no creen tener VIH y consideran el diagnóstico una mentira o lo relacionan con "daño", estas ideas las disuaden de aceptar las indicaciones médicas, afectando la credibilidad y confianza hacia los servicios de salud. Además, piensan que sus hijos inevitablemente nacerán enfermos y morirán pronto, por lo que consideran la cesárea y el cuidado del niño un esfuerzo vano. A estas barreras se suman las diferencias de idioma y lejanía de los establecimientos de salud; también el temor al juicio y condena moral de su comunidad. Conclusiones. Las medidas para la prevención de la TMI parecen extrañas para las costumbres, valores y creencias de las gestantes/madres, por lo que no son comprendidas ni aceptadas, resultando ineficaces y perjudicando la relación con el personal de salud. Se necesita una mejor comprensión de la cultura y el diseño de intervenciones adecuadas a ésta población.
\end{abstract}

Palabras clave: Transmisión Vertical de Enfermedad Infecciosa; VIH; Mujeres Embarazadas; Población Indígena (Fuente: DeCS BIREME)

\section{PREVENTION OF MATERNAL-CHILD TRANSMISSION OF HIV IN PREGNANT WOMEN AND MOTHERS OF THE AWAJUN AND WAMPIS COMMUNITIES IN THE AMAZON REGION OF PERU}

\begin{abstract}
Objectives. To identify the barriers that limit compliance with the prevention of mother-to-child transmission (PMTCT) of HIV measures in two indigenous communities of the Amazon region of Peru. Materials and methods. Qualitative study with a phenomenological approach. Semi-structured interviews were conducted with pregnant women and mothers of children younger than 1 year of the awajún and wampis indigenous communities diagnosed with HIV in the period 2014-2015. Results. The study sample included 15 of 29 eligible women and $87 \%$ were Awajún. Limitations and possible negative effects were observed in cases in which sanitary measures for PMTCT were imposed. Considering their knowledge of diseases, including the presence of symptoms and disabilities, many women from these communities do not believe they are infected with HIV and consider the diagnosis a lie or relate the diagnosis to harm, and these beliefs limit their compliance with medical indications and decrease their trust in health services. In addition, the women believe that their children will inevitably be born sick and will die soon, and thus consider cesarean sections and child care a futile effort. Other factors influencing the successful implementation of PMTCT measures include language barriers, remoteness of health centers, and the fear of judgment and moral condemnation by the local community. Conclusions. PMTCT measures challenge the customs, values, and beliefs of pregnant women and mothers in the Awajún and Wampis indigenous communities, and thus these measures are not understood or accepted, jeopardizing the relationship of the mothers with health personnel. Therefore, cultural factors and interventions appropriate to this population must be better understood.
\end{abstract}

Palabras clave: Transmisión Vertical de Enfermedad Infecciosa; VIH; Mujeres Embarazadas; Población Indígena. (Source: MeSH NLM).

\footnotetext{
Estrategia Sanitaria de Prevención y Control de ITS, VIH/SIDA y hepatitis B. Ministerio de Salud. Lima, Perú.

Instituto Nacional de Salud. Lima, Perú.

Hospital Nacional Arzobispo Loayza. Lima, Perú.

UNICEF, Perú.

a Licenciada, magíster en Epidemiología; ${ }^{\mathrm{b}}$ médico, magíster en salud pública; ${ }^{\mathrm{c}}$ médico cirujano; ${ }^{\mathrm{d}}$ licenciado magíster en salud pública. Recibido: 23/03/2017 Aprobado: 30/11/2017 En línea: 07/12/2017
}

Citar como: Huamán B, Gushiken A, Benites C, Quiroz F, Garcia-Fernández L. Prevención de la transmisión materno-infantil del VIH en gestantes y madres awajún y wampis de la región Amazonas en Perú. Rev Peru Med Exp Salud Publica. 2017;34(4):627-32. doi: 10.17843/rpmesp.2017.344.2725 


\section{INTRODUCCIÓN}

Las gestantes con el VIH no tratadas tienen una tasa de transmisión materno infantil (TMI) de 15 a $40 \%{ }^{(1)}$, se estima que el $20 \%$ se producen antes de las 36 semanas, un $50 \%$ en los días previos al parto y el $30 \%$ en el parto, mientras que el riesgo de transmisión por la lactancia materna puede ser de 30 a $40 \%{ }^{(1,2)}$.

Con el fin de evitar la TMI del VIH, los protocolos del Ministerio de Salud del Perú, en base a las recomendaciones internacionales propone el uso de antirretrovirales combinados en la gestante infectada y el recién nacido expuesto, la culminación del embarazo mediante cesárea electiva, así como la alimentación al recién nacido con leche artificial que, aplicados oportunamente, reducen la TMI a menos del $2 \%$, siendo primordial para ello el acceso temprano al control prenatal y el tamizaje para $\mathrm{VIH}^{(3-5)}$.

A través del sistema de notificación del Centro Nacional de Epidemiología, Prevención y Control de Enfermedades (CDC) y el monitoreo operacional de la Estrategia Sanitaria de ITS, VIH/SIDA y Hepatitis B (ESN ITS/VIH/SIDA y HB), se ha identificado a las poblaciones indígenas amazónicas como altamente vulnerables al $\mathrm{VIH}{ }^{(6,7)}$.

En la Amazonía peruana existen 11 familias lingüísticas que se agrupan en 42 pueblos indígenas ${ }^{(8)}$. El $68 \%$ de los pueblos indígenas amazónicos se concentran en las regiones de Loreto, Amazonas y Ucayali ${ }^{(8)}$. La región Amazonas está habitada por comunidades indígenas de las etnias awajún y wampis, asentadas en la frontera entre Perú y Ecuador, específicamente en la provincia de Condorcanqui y en el distrito de Imaza, donde representan, respectivamente, el 75 y $20 \%$ de la población ${ }^{(9)}$.

Para el periodo 1990-2015, la región Amazonas notificó un total de 684 casos de $\mathrm{VIH}$ (277 mujeres y 407 hombres), de los cuales, $39,5 \%$ fueron reportados por la Red de Salud Condorcanqui y $23,0 \%$ por la MicroRed Imaza, territorios que concentran a las comunidades indígenas awajún y wampis de la región. Cabe señalar, que el 70\% (479) de los casos de VIH fueron notificados en el periodo 2012-2015 probablemente por el mayor acceso a la prueba de $\mathrm{VIH}^{(7,10,11)}$.

La introducción del VIH en los awajún y wampis resultan de las relaciones con las áreas urbanas, como el intercambio comercial, además de patrones de asentamiento de los pueblos indígenas en condiciones de hacinamiento, nuevas actividades económicas, que sumado a los mecanismos internos de salud indígena son inadecuadamente atendidos por el sector salud ${ }^{(12)}$. Aunque la prevalencia del VIH en general es baja, se han documentado poblaciones con elevada prevalencia como es el caso de los chayahuitas $(7,5 \%)^{(13-16)}$.

\section{MENSAJES CLAVE}

Motivación para realizar el estudio. La investigación se propuso debido al aumento de casos de infección por VIH en la población Awajún, sobre todo en gestantes, y a las dificultades para la aplicación de los protocolos de prevención de la transmisión materno-infantil de VIH en éstas comunidades indígenas amazónicas.

Principales hallazgos. Las medidas de prevención de la TMI del VIH por extrañas a la concepción del proceso salud-enfermedad y a las costumbres, valores y creencias de la población awajún y wampis, no son comprendidas ni aceptadas por ellas, resultando ineficaces y, peor, perjudicando la confianza y credibilidad de los servicios de salud.

Implicancias. En salud pública y/o políticas sanitarias. Conocer las concepciones que tiene la población sobre la infección y las medidas de prevención puede contribuir a orientar los mensajes y las políticas públicas en torno a la prevención y control de la enfermedad.

Una revisión de la problemática de gestantes indígenas viviendo con el $\mathrm{VIH}$, evidencia la poca información acerca de prácticas de cuidado y prevención de la TMI, así como la necesidad de un enfoque médico antropológico para atender a las gestantes y mujeres con $\mathrm{VIH}{ }^{(17)}$. Así, este estudio tuvo como objetivo identificar y contribuir con la compresión de las barreras que impiden el cumplimiento de las medidas preventivas de la TMI, dispuestas por el MINSA, en gestantes/madres con VIH procedentes de comunidades indígenas amazónicas de la provincia de Condorcanqui y el distrito de Imaza, en la región Amazonas.

\section{MATERIALES Y MÉTODOS}

\section{DISEÑO Y POBLACIÓN DE ESTUDIO}

Se realizó una investigación cualitativa que buscó, de manera inductiva y con un enfoque fenomenológico ${ }^{(18)}$, identificar las representaciones sociales que tienen las mujeres de las etnias awajún y wampis, en particular las gestantes y madres de niños menores de un año viviendo con el VIH, acerca del VIH/SIDA con el fin de comprender los factores que les impiden cumplir las medidas propuestas por el Ministerio de Salud del Perú para prevenir la TMI.

Se incluyó a todas las mujeres gestantes y madres de niños menores de un año, pertenecientes a las comunidades indígenas de la provincia de Condorcanqui y del distrito de Imaza, diagnosticadas con VIH durante el período 2014-2015, y que fueran mayores de 18 años, según los registros de las Redes de Salud de Condorcanqui y Bagua (MicroRed Imaza).

Se identificaron a 29 gestantes que cumplieron con los criterios de inclusión: diez procedentes de Imaza y 19 de Condorcanqui. De ellas, se tuvo acceso a 20 historias clínicas, encontrando que: 18 pertenecían a la etnia awajún, una a la wampis y una era mestiza. Del total de gestantes, 
se excluyó al caso que correspondía a una mujer mestiza y se logró entrevistar a 15 gestantes/madres diagnosticadas con $\mathrm{VIH}$ de ambas redes de salud.

\section{PROCEDIMIENTOS}

El recojo de información fue realizado a partir de entrevistas semiestructuradas, entre marzo y abril de 2016, por personal de campo femenino con experiencia previa en la zona, el cual fue capacitado por el investigador miembro del equipo con experiencia en estudios cualitativos. Asimismo, se contó con un intérprete local quien participó en aquellas entrevistas en las que las mujeres no entendían ni hablaban español. Además, se elaboró previamente una guía de entrevistas a partir de la cual se indagó sobre las experiencias de las gestantes/madres respecto a la atención en los servicios de salud, sus conocimientos y representaciones sobre el $\mathrm{VIH} / \mathrm{SIDA}$, las vivencias generadas al recibir el diagnóstico de la infección, su experiencia del embarazo, y sus representaciones y comportamientos con relación al tratamiento de la infección y a las medidas de prevención de la TMI (en particular, la decisión de tener el parto por cesárea y no dar lactancia materna a sus hijos).

Para las entrevistas, las mujeres fueron invitadas a participar voluntariamente a través de los establecimientos de salud de la zona, donde también realizaron los controles de su salud y la de sus hijos, en algunos casos cuando no podían llegar hasta el establecimiento de salud fueron visitadas en sus domicilios. Todas las entrevistas se dieron en un espacio privado, ya sea en un consultorio facilitado por el establecimiento o en algunos casos en su domicilio, donde solo estuvo presente la entrevistadora y la participante, cuando la madre/gestante no hablaba español ingresó el traductor (casos). Las entrevistas fueron grabadas, previa explicación y consentimiento informado de las participantes.

Las grabaciones fueron transcritas, identificando las unidades hermenéuticas, que fueron posteriormente codificadas y agrupadas por afinidad temática. Establecidas las categorías emergentes, se determinó las relaciones existentes entre ellas, en especial aquellas que permitieran comprender las barreras culturales que impiden el cumplimiento de las medidas de prevención de la TMI del $\mathrm{VIH}$ en las comunidades indígenas. Para el análisis, se utilizó el programa de análisis de datos cualitativos Atlasti versión 7 y estuvo a cargo de un experto en estudios cualitativos.

\section{CONSIDERACIONES ÉTICAS}

El protocolo del estudio fue aprobado por el Comité de Ética Institucional y de Investigación del Hospital Nacional MadreNiño San Bartolomé. Cada participante recibió información verbal y escrita sobre los objetivos del estudio, así como las condiciones para la entrevista, lo cual fue traducido a su idioma cuando fue necesario. Fue asegurada la privacidad, además de la confidencialidad en el manejo de los audios y datos. Para garantizar el anonimato, los nombres fueron sustituidos por códigos alfanuméricos.

\section{RESULTADOS}

\section{CARACTERISSTICAS DE LAS PARTICIPANTES}

De las gestantes/madres entrevistadas; según sus características sociodemográficas, once eran multíparas $(11 / 15)$ y dos eran gestantes (2/15), con una edad promedio de 24,2 años. Diez se enteraron de su diagnóstico durante el embarazo, dos durante el trabajo de parto y tres siendo puérperas, de ellas cinco recibieron tratamiento antirretroviral durante la gestación y once tuvieron parto vaginal. Se encontraron datos de 10 niños expuestos al VIH, tres de los cuales fallecieron, cuatro recibieron profilaxis antirretroviral y nueve recibieron lactancia materna.

\section{REPRESENTACIONES Y VIVENCIAS SOBRE EL VIH/ $S I D A$}

En general, las gestantes/madres cuentan con información básica sobre el VIH/SIDA. Sin embargo, resulta limitada en relación a sus síntomas, de cómo estos evolucionan en el tiempo, sus causas y medidas de prevención: En ese tiempo yo sabía que el VIH no tiene cura, se mueren, se hacen flaquitos y no tienen cura solamente tratamiento algo asi (E01). Otra madre: Cuando tenemos este virus del VIH se enflaquece, sale heridas, además no tiene cura, eso nomás escuché (E05).

La información fue brindada al momento del tamizaje y luego del diagnóstico, antes su conocimiento era mínimo o nulo. De allí que, cuando éste les fue entregado, en la mayoría de los casos, sintieron miedo ante lo que se presenta como la inminencia de la muerte: Me quedé, no sé, fría, me quedé con susto, me quedé preocupada más que todo. [...] Porque me han dicho que no tiene cura, que esa enfermedad no tiene cura, más que todo por eso, más mi hijito me preocupaba bastante, el mayorcito (E08).

El tener VIH, a veces constituye en fuente de culpa y un elemento empleado como medio de coacción y chantaje: [Mi exesposo] me dijo todo voy a contar en Pacuna cómo eres', y me dijo 'y todos se van a asustar y nadie te va sacar, nadie se va acercar, nadie te va tratar bonito', me dijo así. Una vez vino a Pacuna, llamó a mi tío y a mi tía a decirles que yo tenía VIH/SIDA, que tenía todo, bueno, todito le explicó (E01). Como consecuencia, las mujeres no acuden a los servicios por temor a que se sepa de su infección: $L a$ población está que averigua, dice que chismea qué es lo que tiene, de dónde viene el virus... (E04). 
Tanto los mensajes que reciben, como los acontecimientos que enfrentan, son interpretados en función de las concepciones y representaciones con las que cuentan. El traductor se refiere a la entrevistada: No tiene nada, no tiene síntoma, malestar tampoco, ella no se siente mal, ella dice 'de repente me están diciendo mentira' o ‘cómo puede tener enfermedad?', como dice, duda, '¿tendré o no tendré?', dice. [...] Ella lo que dice que tiene es por brujo, porque ella no enferma, siempre sale a jugar vóley, se va a pescar, a la chacra, no tiene ni un síntoma, esa enfermedad que no tiene síntoma (E11).

\section{PREVENCIÓN DE LA TRANSMISIÓN MATERNO- INFANTIL}

Por otra parte, respecto a las medidas para evitar la TMI, creen que inevitablemente sus hijos nacerán enfermos y morirán: De repente yo me opero señorita, digo se va a morir mi hijo, de repente obligado me van a hacer cesárea, en vano va a vivir y me van a cesarear, o sea, con mi hijo, me van a cesarear $y$, de todas maneras, se va morir, de repente mi cesárea es en vano [...]. Si me hago cesárea, en vano voy a sufrir. Si mi hijo va a vivir todo el tiempo, bueno sería, pero en vano me voy a hacer, digo yo, [...] que me corten mi barriga, que me corten, que me saquen mi bebé, de repente me rajan y, bueno, me cortan, me operan y mi hijito de todas maneras va morir (E01). Además, existe influencia de las madres de las gestantes en las decisiones, que muchas veces es diferente a lo indicado por el personal de salud: Me han dicho para hacer cesárea, pero yo di en mi casa porque mi mamá no me ha dejado, 'no quiero que te corten', me decía... (E08). En otros casos, cuestionan que las imposibilita de realizar sus labores domésticas, cuidado de sus hijos y trabajos en la chacra: Porque awajún carga yuca, como cantidad [...], en una canasta, ¿no? y eso no podía cargar y no podía caminar lejos (E05).

Una objeción que pudiera encontrarse a la base de la resistencia a no dar lactancia materna, es su valoración de la misma, que las lleva a cuestionar la indicación: ¡¿Cómo no va a dar, pobrecito, leche?, ¿cómo no va a dar, así le den otra leche, no va a dar teta?! (E03). Además, manifiestan: Mi mamá, mi cuñada, siempre me han dicho que, de todas maneras, siempre el bebito está contagiado, siempre así, tu hijo está dentro de tu vientre, es así, tienes que darles de mamar pues, porque, bueno, hasta que llegue su [...], ¿cómo se llama?, su hora, para que no le hagas sufrir de hambre, me han dicho mis cuñadas (E08).

\section{ACCESO Y ADHERENCIA AL TRATAMIENTO}

Se encontró barreras para la aceptación y adherencia del tratamiento: Porque no entiende el castellano y más que no tenía plata [...] Dice que cuando vaya a recibir tratamiento no puede irse ni salir de acá. [...] Es que tiene duda, no quiere ¿no? Y si trae medicamento [es mejor] a que se vaya ella en persona, no se puede, no quiere, cuando trae medicamento, dice que así, se puede tomar [...] (E11).

Este rechazo también se asocia con la incredulidad sobre el diagnóstico: El técnico le ofreció el medicamento que estaba allá en el puesto y ella rechazaba porque no estaba segura de su enfermedad. Quiere ir personalmente a un centro de salud para que puedan sacar sangre y puedan de nuevo hacer el análisis [...]. Cuando sale positivo del bebé, ella también va ya tomar en serio este problema y va empezar el tratamiento (E06).

Los eventos adversos que genera la medicación también constituyen un problema para ellas: Las pastillas da dolor de cabeza y malestar y mareo, es muy fuerte, icómo lo voy a soportar!, [... .] cuando uno se levanta temprano, uno ya se muere, duele la cabeza, ya no se puede ni irse a trabajar, ¿quién, señorita, me va a cocinar, me va a dar de comer? [...]. Entonces, nosotros estamos en tratamiento, pero peor me mata eso; me venía el dolor de cabeza, me despertaba borracha, ya no podía caminar (E01).

Las mujeres entrevistadas manifestaron su preferencia por el uso de plantas medicinales para tratar las enfermedades, entre ellas, el VIH: Con plantas medicinales, hay que ver, de repente, que se puede sanar (E05). Un esposo dijo a la gestante: Por lo que estás tomando, pastillas, ya lo has matado (al bebé) y puedo tener un problema grande. Dejé de tomar, y bien tranquilo mi hijo ha crecido, se mueve, zapatea. Vuelta intenté tomar, y ya no podía (E01).

Finalmente, que el tratamiento no cure y, por el contrario, genere efectos secundarios, pone en cuestionamiento el propio diagnóstico y los conocimientos del personal de salud: Como cuenta, sentía malestar, dolor de cabeza, náuseas y todo, entonces, sus papás le dijeron que deje de tomar, que ellos la van a llevar a otro particular, al curandero, para que el curandero le diga si realmente tiene el virus o es daño (E04).

\section{DISCUSIÓN}

El estudio muestra, a propósito del abordaje de la TMI de la infección por $\mathrm{VIH}$, las limitaciones y las posibles dificultades para los servicios de salud de impulsar un tratamiento y medidas preventivas que, por extrañas a la cosmovisión (es decir extrañas a sus costumbres, valores y creencias) y particularmente a las concepciones del proceso saludenfermedad de las poblaciones indígenas, no logran ser comprendidas y aceptadas. En este caso, tratándose de la prevención de la TMI del VIH, se indica: tamizaje de VIH en todas las gestantes $\mathrm{y}$, a aquellas que resultan positivas, se propone iniciar el tratamiento antirretroviral, parto por cesárea y suspender la lactancia materna en cumplimiento con el protocolo del Ministerio de Salud ${ }^{(5)}$. 
Según las entrevistas, los efectos son diversos, las mujeres no comprenden o desconfían del diagnóstico, tampoco comprenden los argumentos que les dan para adoptar un tratamiento de por vida y las demás medidas de prevención de TMI (en especial, la cesárea).

En consecuencia, las mujeres no reciben el tratamiento y no acuden a los establecimientos de salud para la atención del parto, principalmente por el temor de que las operen. De manera similar, un estudio realizado en Brasil (Ribeirao Preto), sobre las creencias de las madres con VIH respecto a la transmisión vertical, encontró que el conocimiento no siempre modifica el comportamiento, más aún si no se cree en la existencia del virus ${ }^{(19)}$.

En los casos que aceptan recibir antirretrovirales, los eventos adversos, que muchas veces resultan ser incapacitantes, las convencen (y a sus familias) rápidamente de abandonarlo, con lo cual se alivian y confirman que era el tratamiento lo que las enfermaba, así como a sus hijos.

En este contexto -el generado por un diagnóstico no creíble, sin síntomas ni enfermedad en curso, y por un tratamiento que enferma e incapacita-, resulta afectada la credibilidad de los servicios de salud y del personal que atiende, que caen bajo sospecha de no saber o que "de repente, me están diciendo mentiras", profundizando la brecha en la comunicación y la confianza entre ellos y la población.

Por otra parte, se ha transmitido la idea de que la "enfermedad" es mortal y que no tiene cura, y que, teniendo la madre el diagnóstico de infección por $\mathrm{VIH}$, el hijo en su vientre nacerá indefectiblemente enfermo y pronto va a morir, con lo cual deja de ser sujeto privilegiado de atención y cuidado volviéndose susceptible de abandono.

Frente a los resultados encontrados, las medidas para la prevención de la TMI resultan no solo ineficaces, sino desfavorables, para la relación entre la población (y por ende los pacientes) awajún y wampis y los servicios de salud en la zona, pudiendo afectar al conjunto de programas de salud que estos brindan. Cabe preguntarse qué se puede hacer, incluso si conviene sostener las acciones del programa o detenerse para una mejor comprensión del problema y diseñar una intervención adecuada a las comunidades indígenas amazónicas.

En concordancia a lo descrito anteriormente, resulta importante indagar y conocer cómo conciben los pobladores "la enfermedad" y los modos de transmisión, cómo afecta a las personas provocando su empeoramiento o la muerte, cómo intervienen sus agentes sanitarios, cómo actúan los tratamientos frente a las enfermedades, y aprender de ellos, ya no solo para implementar las medidas de prevención de la TMI, sino otros programas de salud.
Preguntar por las creencias y la experiencia de la población permitirá identificar elementos de su concepción que sirvan como puntos de apoyo para explicar la visión que tiene la ciencia acerca de las enfermedades y sus causas. Por lo tanto, debiera permitirse la elaboración de contenidos y metodologías con los cuales se procure la transmisión de dicha visión en las actividades que realizan los servicios de salud. Es básico entonces, reconocer la necesidad de entender no solo a la gestante/madre sino el entorno en el que vive ${ }^{(20)}$

En tal sentido, la pregunta y escucha constantes de las ideas y experiencias de la población pueden ser de gran utilidad para entender las significaciones y los efectos que van adquiriendo los mensajes proporcionados por los servicios. Esto permitirá afinar o rectificar los mismos, pero más importante aun, permitirán acoger los decires y saberes, las creencias y experiencias de las gestantes/ madres indígenas. Además, se logrará reducir las brechas de comunicación e incorporar la búsqueda de soluciones -en principio, seguramente propias y, luego, tal vez importadas. De este modo, se irá consiguiendo la articulación entre el sistema de salud oficial y el sistema de salud propio de los pueblos indígenas, allí donde hoy se levantan barreras que tienen por cimiento la percepción de rechazo a su cultura ${ }^{(21)}$.

El estudio tuvo limitaciones en los casos en que fue necesario la intervención del intérprete dado que las respuestas no siempre pudieron ser totalmente espontáneas. Cabe mencionar que los resultados del estudio sólo corresponden a las mujeres indígenas de la región Amazonas y no se pueden generalizar a todas las mujeres awajún y wampis de otras regiones, ni a otras etnias de la Amazonía.

En conclusión, el estudio ha permitido identificar las limitaciones y los posibles efectos de imponer los conocimientos sobre el $\mathrm{VIH}$ y medidas sanitarias para la prevención de la TMl por parte del sector salud. Las medidas sanitarias son extrañas a las costumbres, valores y creencias $\mathrm{y}$, particularmente, a la concepción del proceso salud-enfermedad de las poblaciones indígenas, por ello son comprendidas y aceptadas por las comunidades, resultando no solo ineficaces sino perjudiciales, para la relación entre la población y los servicios de salud. Ésta situación mella la credibilidad y confianza de estos últimos, y afecta el conjunto de programas de salud que se brindan.

En este contexto, resulta importante preguntar si conviene, o no, mantener las acciones del programa o detenerse para buscar una mejor comprensión del problema y un diseño de intervención adecuado a las condiciones culturales de la población a la cual se dirige el Ministerio de Salud.

Agradecimientos: al equipo de trabajo Helen Palma y Rosa Acosta, a Víctor Melt Campos por la revisión y comentarios. También a los Responsables de VIH de Amazonas: Roxana Cubas (DIRESAAmazonas), Jenny Oliva (Red de Condorcanqui) 
y Norvil Cabrera (Red de Salud Bagua), por las coordinaciones y facilidades para la realización del estudio.

Fuentes de financiamiento: este trabajo fue financiado por la UNICEF Perú.

Conflictos de interés: los autores declaran no tener conflictos de interés. Las opiniones expresadas aquí son responsabilidad de los autores.
Contribuciones de los autores: BH participó en la elaboración del protocolo, coordinación del estudio en campo, concepción y diseño del estudio, recolección, análisis e interpretación de datos y redacción del artículo. AG participó en la elaboración del protocolo, concepción y diseño del estudio, análisis e interpretación de datos y redacción del artículo. CB participó en la asesoría técnica en el desarrollo del estudio, revisión y redacción del artículo. FQ participó en la recolección de resultados y obtención de financiamiento. LGF participó en el análisis e interpretación de datos, revisión y redacción del artículo.

\section{REFERENCIAS BIBLIOGRÁFICAS}

1. Connor EM, Sperling RS, Gelber R, Kiselev P, Scott G, O'Sullivan MJ, et al. Reduction of maternal-infant transmission of human immunodeficiency virus type 1 with zidovudine treatment. Pediatric AIDS Clinical Trials Group Protocol 076 Study Group. N Engl J Med. 1994 Nov 3;331(18):1173-80.

2. Kourtis AP, Bulterys M, Nesheim SR, Lee FK. Understanding the timing of HIV transmission from mother to infant. JAMA. 2001 Feb 14;285(6):709-12.

3. Cooper ER, Charurat M, Mofenson L, Hanson IC, Pitt J, Diaz C, et al. Combination antiretroviral strategies for the treatment of pregnant HIV-1-infected women and prevention of perinatal HIV-1 transmission. J Acquir Immune Defic Syndr. 2002;29(5):484-94.

4. World Health Organization. Consolidated guidelines on the use of antiretroviral drugs for treating and preventing HIV infection: recommendations for a public health approach. 2013.

5. Ministerio de Salud. NTS 108-MINSA/ DGSP-V.01 "Norma Técnica de Salud para la Prevención dela Transmisión madre-niño del VIH y la Sífilis”. Perú, 2014.

6. Pathfinder International. Informe Subvención Fondo Mundial: Expansión de la Respuesta Nacional al VIH en Poblaciones Clave y Vulnerables de Ámbitos Urbanos y Amazónicos del Perú. Perú, 2016.

7. Dirección General de Epidemiología. Situación del VIH/SIDA en el Perú. Bo- letín Epidemiológico Mensual [Internet]. Lima; 2016 Dec [cited 2016 Oct 17]. Available from:

http://www.dge.gob.pe/portal/index. php?option $=$ com_content $\&$ view $=$ article\&id $=362 \&$ Itemid $=362$

8. Ministerio de Cultura. Base de Datos de Pueblos Indígenas u Originarios [Internet]. [cited 2016 Nov 2]. Available from: http://bdpi.cultura.gob.pe/

9. Instituto Nacional de Estadística e Informática. II Censo de comunidades indígenas de la Amazonia peruana 2007. Lima; 2008.

10. Instituto Nacional de Salud. NetLab [Internet]. [cited 2016 Oct 18]. Available from: https://www.netlab.ins.gob.pe/ FrmNewLogin.aspx

11. Dirección de Salud de Amazonas. Reporte de casos de VIH/SIDA en Amazonas, periodo 2015-2016.

12. Asociación Interétnica de Desarrollo de la Selva Peruana - AIDESEP. Sistemas de Salud Indígena. Lima; 2010.

13. Ormaeche M, Whittembury A, Pun M, Suárez-Ognio L. Hepatitis B virus, syphilis and HIV seroprevalence in pregnant woman and their male partners from six indigenous populations of the Peruvian Amazon, 2007-2008. Int J Infect Dis. 2012 Oct;16(10):e724-30. doi: 10.1016/j. ijid.2012.05.1032.

14. Zavaleta C, Fernández C, Konda K, Valderrama Y, Vermud SH, Gotuzzo E. High prevalence of HIV and syphilis in a remote native community of the Peruvian Amazon. Am J Trop Med Hyg. 2007;76(4):703-5.

15. PAHO. A systematic review of the literature on HIV, Sexually Transmitted Infections, and viral hepatitis among indigenous, afro-descendant and Romani populations in Latin America (2000-2016): An emphasis on the burden of disease and Knowledge, Attitudes and Practices (KAP), 2016.

16. UNICEF. Síntesis de evidencia de la situación de salud de la población indígena y amazónica de Perú y Bolivia, con énfasis en ITS y VIH y Hepatitis B. Lima, 2014.

17. Portocarrero J. VIH en gestantes indígenas: un reto para la salud pública peruana. Rev Perú Med Exp Salud Pública. 2015 JulSep;32(3):546-54.

18. Lévano AC. Investigación cualitativa: diseños, evaluación del rigor metodológico y retos. Liberabit. 2007;13(13):71-8.

19. Neves LA de S, Gir E. Creencias de las madres seropositivas respecto a la transmisión vertcial del VIH. Rev Lat-Am Enferm. 2006;14(5):781-788.

20. Vidal L, Loû A. AIDS and social situations of women in Africa: from notions to research methods. Sante, 2001;11(4):265-72.

21. Defensoría del Pueblo. La defensa del derecho de los pueblos indígenas amazónicos a una salud intercultural. Lima, Perú; 2015.

Correspondencia: Byelca Huamán

Dirección: Av. Horacio Urteaga 900, Jesús

María, Lima, Perú

Teléfono: 511956016955

Correo electrónico:byelca@gmail.com 